\title{
Magnetic properties of lithium rare-earth fluorides: Ferromagnetism in LiErF4 and LiHoF4 and crystal-field parameters at the rare-earth and Li sites
}

\author{
Hansen, P. E.; Johansson, Torben; Nevald, Rolf
}

Published in:

Physical Review B

Link to article, DOI:

10.1103/PhysRevB.12.5315

Publication date:

1975

Document Version

Publisher's PDF, also known as Version of record

Link back to DTU Orbit

Citation (APA):

Hansen, P. E., Johansson, T., \& Nevald, R. (1975). Magnetic properties of lithium rare-earth fluorides:

Ferromagnetism in LiErF4 and LiHoF4 and crystal-field parameters at the rare-earth and Li sites. Physical Review B, 12(11), 5315-5324. https://doi.org/10.1103/PhysRevB.12.5315

\section{General rights}

Copyright and moral rights for the publications made accessible in the public portal are retained by the authors and/or other copyright owners and it is a condition of accessing publications that users recognise and abide by the legal requirements associated with these rights.

- Users may download and print one copy of any publication from the public portal for the purpose of private study or research.

- You may not further distribute the material or use it for any profit-making activity or commercial gain

- You may freely distribute the URL identifying the publication in the public portal 


\title{
Magnetic properties of lithium rare-earth fluorides: Ferromagnetism in $\mathrm{LiErF}_{4}$ and $\mathrm{LiHoF}_{4}$ and crystal-field parameters at the rare-earth and $\mathrm{Li}$ sites*
}

\author{
P. E. Hansen, T. Johansson, and R. Nevald \\ Department of Electrophysics, The Technical University of Denmark, DK-2800 Lyngby, Denmark \\ (Received 23 June 1975)
}

\begin{abstract}
Single crystals of $\mathrm{LiErF}_{4}$ and $\mathrm{LiHoF}_{4}$ have been grown and their magnetic properties measured from $1.3 \mathrm{~K}$ to $300 \mathrm{~K}$. $\mathrm{LiHoF}_{4}$ turned out to be a nearly ideal Ising ferromagnet with $T_{C}=1.30 \pm 0.05 \mathrm{~K}$ and a saturation magnetization along the crystalline $c$ axis of $(6.98 \pm 0.02) \mu_{B} . \operatorname{In} \mathrm{LiErF}_{4}$ no ordering was observed, but extrapolation indicates that below $0.5 \mathrm{~K}$ it will be ferromagnetic with the magnetic moments in the crytalline $a b$ plane. From the susceptibilities the crystal-field parameters $B_{n}^{m}$ with $(n, m)=(2,0),(4.0),(4,4),(6,0),(6,4)$ have been extracted giving for $\mathrm{Er}^{3+}$ in $\mathrm{LiErF}_{4}$ : 430., $-985 ., 1185 .,-5 ., 740 .+i 135 .\left(\mathrm{cm}^{-1}\right)$ and for $\mathrm{Ho}^{3+}$ in $\mathrm{LiHoF}_{4}: 470 .,-825 ., 1050 .,-10 ., 760 .+i 150\left(\mathrm{~cm}^{-1}\right)$. The exchange constants were found to be small compared to the dipole interactions. Furthermore the ${ }^{7} \mathrm{Li}$ NMR spectra have been obtained in these materials as well as in $\mathrm{LiTbF}_{4}$ thereby determining the second-order crystal-field parameters at the Li site: $q=\mathbf{0 . 0 3 6 3}$, 0.0354 , and $0.0343 \AA^{-3}$ for the $\mathrm{Er}, \mathrm{Ho}$, and $\mathrm{Tb}$ compound, respectively. The crystal-field parameters are compared to values calculated from point charges, showing agreement at the $\mathrm{Li}$ sites but not at the rare earth sites.
\end{abstract}

\section{INTRODUCTION}

Most $\mathrm{Li} R_{x} \mathrm{Y}_{1-x} \mathrm{~F}_{4}$, where $R$ is a rare earth, crystallize in the tetragonal scheelite structure $I 4_{1} / a$ for any value of $x .^{1}$ They form a group of materials which are interesting from a practical as well as a theoretical point of view. They are efficient laser materials ${ }^{2}$ and can be used for frequency conversion in the infrared-visible region. ${ }^{3}$ The theoretical attraction of these materials stems from their simplicity. They consist of fairly ionic atoms, possess rather high point symmetry $\left(S_{4}\right.$-almost $\left.D_{2 d}\right)$ through the rare earth, and have the rare earths mutually coupled primarily through dipole forces.

Many types of experimental data have been accumulated for various compounds in this group. Most work has been done on the $\mathrm{LiYF}_{4}$ dilutely doped with rare-earth ions. Absorption and fluorescence spectra have been measured on $\mathrm{LiTm}_{0.02} \mathrm{Y}_{0.98} \mathrm{~F}_{4}$ by Jenssen et al. , ${ }^{4}$ on $\mathrm{LiNd}_{0.02} \mathrm{Y}_{0.98} \mathrm{~F}_{4}$, $\mathrm{LiYb}_{0.02} \mathrm{Y}_{0.98} \mathrm{~F}_{4}$, and $\mathrm{LiNd}_{0.02} \mathrm{Yb}_{0.02} \mathrm{Y}_{0.96} \mathrm{~F}_{4}$ by Miller and Sharp, ${ }^{5}$ on $\operatorname{LiNd}_{\epsilon} \mathrm{Y}_{1-\epsilon} \mathrm{F}_{4}$, where $\epsilon=2.2,1.4$, and $0.24 \%$, by Harmer et al. , ${ }^{6}$ and on $\mathrm{LiYF}_{4}$ doped with $1 \%$ and $3 \% \mathrm{Er}$ by Brown et al. ${ }^{7}$ ESR spectra have been obtained from traces of $\mathrm{Nd}^{3+}, \mathrm{Dy}^{3+}$, $\mathrm{Er}^{3+}$, and $\mathrm{Yb}^{3+}$ in $\mathrm{LiYF}_{4}$ by Sattler and Nemarich ${ }^{8}$ and from $\mathrm{Tb}^{3+}$ in $\mathrm{LiTb}_{0.01} \mathrm{Y}_{0.99} \mathrm{~F}_{4}$ by Laursen and Holmes. ${ }^{9}$ Previous studies on compounds with $100 \%$ rare earth have concentrated on $\mathrm{LiTbF}_{4}$. Holmes et al. have determined the bulk susceptibility, Curie point, and spontaneous magnetization through macroscopic measurements, ${ }^{10}$ and crystal-field excitations through neutron studies. ${ }^{11}$ Als-Nielsen et al. also determined the wave-vector-dependent susceptibility. ${ }^{12}$

There is probably a good chance that the be- havior of rare earths in ionic crystals can be understood, or at least some general empirical rules for that behavior be established, by a manyaspect study of this group. We are therefore extending the list of materials under investigation to the dense crystals $\mathrm{Li} R \mathrm{~F}_{4}$, where $R=\mathrm{Gd}$, Dy, Ho, Er, Tm, Yb, and the list of applied experimental methods to include $\mathrm{Li}$ and $\mathrm{F}$ nuclear magnetic resonance.

Here we report on the magnetic data and the crystal-field parameters (CFP) at the rare-earth and $\mathrm{Li}$ sites in $\mathrm{LiErF}_{4}, \mathrm{LiHoF}_{4}$, and $\mathrm{LiTbF}_{4}$. The rare-earth CFP's are derived from susceptibility and magnetization measurements, and the Li CFP's from ${ }^{7} \mathrm{Li}$ NMR.

In future work the transferred hyperfine interactions between the $R$ and the $\mathrm{Li}$ and $\mathrm{F}$ nuclei, as well as the $R$ spin relaxation, will be studied by NMR on the Li and F nuclei.

\section{THEORY}

\section{A. Crystal field on rare-earth ions}

For the ground level $|L S J\rangle$ and in the meanfield approximation the Hamiltonian for a trivalent rare-earth ion in $\mathrm{Li} R \mathrm{~F}_{4}$ with an inner magnetic field $\overrightarrow{\mathrm{H}}_{\text {in }}$ can be written

$$
\begin{aligned}
\mathcal{H}_{R} & =\mathcal{H}_{\mathrm{cr}}+\mathcal{H}_{\text {magn }} \\
& =\sum_{i=2,4,6} \beta_{J}^{i} \sum_{m=0}^{i} B_{i}^{m} O_{i}^{m}(\overrightarrow{\mathrm{J}})-g_{J} \mu_{B} \overrightarrow{\mathrm{J}} \cdot \overrightarrow{\mathrm{D}} \cdot \overrightarrow{\mathrm{H}}_{\mathrm{in}},
\end{aligned}
$$

where $O_{i}^{m}$ are the Stevens operators (Hutching ${ }^{13}$ ), $B_{i}^{m}$ are the CFP's, $\beta_{J}^{i}$ are the Stevens multiplicative factors (usually called $\alpha_{J}, \beta_{J}, \gamma_{J}$ ), $g_{J}$ the Lande factor, $\mu_{B}$ the Bohr magneton, $\vec{J}$ the dimensionless rare-earth-ion angular momentum, and 
$\overleftrightarrow{\mathrm{D}}$ a tensor transforming the inner field to the local field $\overrightarrow{\mathrm{H}}_{1 \mathrm{oc}}$ at the rare-earth sites.

For a sample in the paramagnetic state

$$
\overrightarrow{\mathrm{H}}_{1 \mathrm{oc}}=\left[1+\left(\frac{4}{3} \pi \rho+\vec{\delta}+\vec{\epsilon}\right) \vec{\chi}\right] \overrightarrow{\mathrm{H}}_{\mathrm{in}},
$$

where $\vec{\chi}$ is the mass susceptibility, $\rho$ the density, and $\vec{\delta}$ the dipole field tensor

$$
\vec{\delta}=\kappa \sum_{i} \frac{3 \overrightarrow{\mathrm{r}}_{i} \overrightarrow{\mathrm{r}}_{i}-\overrightarrow{1}_{i}^{2}}{r_{i}^{5}},
$$

$\kappa$ being the mass per formula unit and $\overrightarrow{\mathrm{r}}_{i}$ the vector to the $i$ th rare-earth neighbor. $\vec{\epsilon}$ takes into account possible exchange interactions. $(\vec{\delta}+\vec{\epsilon}) \chi \vec{H}_{i n}$ thus is the effective magnetic field arising from the rare-earth moments in a large sphere around the rare earth. The numerical values of the $\vec{\delta} c$ components for summation over spheres consisting of $\sim 900$ and 5000 dipoles, respectively, are given in Table I. It is seen that convergence is achieved. In a coordinate system with the $z$ axis along the fourfold axis, the $S_{4}$ symmetry allows seven CFP's: $B_{2}^{0}, B_{4}^{0}, \operatorname{Re} B_{4}^{4}, \operatorname{Im} B_{4}^{4}, B_{6}^{0}, \operatorname{Re} B_{6}^{4}$, and $\operatorname{Im} B_{6}^{4}$, three elements in $\vec{\delta}: \delta_{x x}=\delta_{y y}=-\frac{1}{2} \delta_{z z}\left(=-\frac{1}{2} \delta_{c}\right)$, three in $\vec{\chi}$ : $\chi_{x x}=\chi_{y y}\left(=\chi_{a}\right), \chi_{z z}\left(=\chi_{c}\right)$ and three in $\overrightarrow{\epsilon:} \epsilon_{x x}=\epsilon_{y y}\left(=\epsilon_{a}\right)$, $\epsilon_{z z}\left(=\epsilon_{c}\right)$. [The two independent elements of $\vec{\epsilon}$ will be called the exchange parameters (EP). ] It is possible to choose the $x$ direction so that $\operatorname{Im} B_{4}^{4}=0$, although low-field susceptibility measurements can not determine this direction in the crystal. We will in the following paragraphs refer to this coordinate system.

As discussed by Jenssen et al. ${ }^{4}$ the point symmetry is nearly $D_{2 d}$, and in this case also $\operatorname{Im} B_{6}^{6} \approx 0$.

If $\mathcal{H}_{\mathrm{cr}}$ is diagonalized giving the eigenfunctions $|n\rangle$ and eigenvalues $E_{n}$, the components of the zero-field magnetic polarizability per ion at temperature $T$ can be obtained according to the formula

$\alpha_{j}(T)=\left[\left(g_{J} \mu_{B}\right)^{2} / \sum_{n} \exp \left(\frac{-E_{n}}{k_{B} T}\right)\right]\left(\sum_{n} \sum_{m}^{\prime}\left|\left\langle n\left|J_{j}\right| m\right\rangle\right|^{2} \frac{\exp \left(-E_{n} / k_{B} T\right)}{k_{B} T}-2 \sum_{n} \sum_{m}^{\prime \prime}\left|\left\langle n\left|J_{j}\right| m\right\rangle\right|^{2} \frac{\exp \left(-E_{n} / k_{B} T\right)}{E_{n}-E_{m}}\right)$,

where $\sum_{m}^{\prime}$ runs over states with $E_{m}=E_{n}$ and $\sum_{m}^{\prime \prime}$ over states with $E_{m} \neq E_{n}$. $k_{B}$ is Boltzmann's constant. is

The relation between the susceptibility $\chi_{j}$ and $\alpha_{j}$

$$
1 / \chi_{j}=\kappa / \alpha_{j}-\left(\frac{4}{3} \pi \rho+\delta_{j}+\epsilon_{j}\right)
$$

By minimizing the relative standard deviation

$S=\left\{\sum_{j=a, c}\left[\sum_{i=1}^{N_{j}}\left(\frac{\chi_{j}^{\mathrm{calc}}\left(T_{i}\right)-\chi_{j}^{\text {expt }}\left(T_{i}\right)}{\chi_{j}^{\text {exp }}\left(T_{i}\right)}\right)^{2}\right] / \sum_{j=a, c} N_{j}-\eta\right\}^{1 / 2}$

with respect to the CFP's and EP's, these quantities can be determined. $N_{j}$ is the number of measured points with the field along the $j$ direction and $\eta$ is the number of parameters to be fitted.

A fit including all possible independent parameters $B_{2}^{0}, B_{4}^{0}, B_{4}^{4}, B_{6}^{0}, \operatorname{Re} B_{6}^{4}, \operatorname{Im} B_{6}^{4}, \epsilon_{a}$, and $\epsilon_{c}$ was performed. Another fit including only $B_{2}^{0}, B_{4}^{0}, B_{4}^{4}$, and $\operatorname{Re} B_{6}^{4}$ was also made, because the first fit showed the remaining parameters to be small compared to their accuracies.

B. Crystal field on the Li ions

The Li site like the rare-earth site has $S_{4}$ symmetry and therefore the ${ }^{7} \mathrm{Li}$ nuclear Hamiltonian is $\mathcal{H}_{\mathrm{L} i}=\mathcal{H}_{\text {masn }}+\mathcal{H}_{\mathrm{cr}}=-\gamma_{\mathrm{Li}} \hbar \overrightarrow{\mathrm{I}} \cdot \overrightarrow{\mathrm{D}} \cdot \overrightarrow{\mathrm{H}}_{\text {in }}+\frac{1}{12}\left[e^{2} Q\left(1+\gamma_{\infty}\right) q\right] O_{2}^{0}(\overrightarrow{\mathrm{I}})$,

where $\vec{I}$ is the nuclear dimensionless angular momentum, $\gamma_{L_{i}}$ the gyromagnetic factor, $e$ the elementary charge, $\overrightarrow{\mathrm{H}}_{\text {in }}$ the inner field, $Q$ the quadrupole moment, and $\gamma_{\infty}$ the antishielding factor.
Finally,

$$
q=\left.\frac{1}{e} \frac{\partial^{2} V_{\mathrm{cr}}}{\partial z^{2}}\right|_{\text {Li site }},
$$

where $V_{\text {cr }}$ is the crystal potential.

Although $\mathcal{F}_{\mathrm{L}_{1}}$ has the same form as $\mathfrak{H}_{R}$ it looks different in three ways: (i) The nuclear parameters are not defined exactly the same way as the ionic ones. (ii) For ${ }^{7} \mathrm{Li}\left(I=\frac{3}{2}\right)$ the effect of the CFP's of order higher than 2 is zero. (iii) $\mathcal{H}_{\text {magn }} \gg \mathcal{H}_{\text {cr }}$ in the nuclear case contrary to the ionic case.

In the Li NMR experiment $\mathcal{H}_{\mathrm{cr}}$ gives rise in first order to a splitting of the spectrum in an unshifted line and two symmetrically positioned side lines. The relative intensity of the center line to any of the side lines is $4: 3$. From the distance between the lines $q$ can be determined. The center of gravity is also shifted owing to the magnetic field from the rare earth. The information obtainable from this shift will be published in a subsequent paper together with similar shifts found for the ${ }^{19} \mathrm{~F}$ nuclei.

TABLE I. $\delta_{c}$ 's calculated by summing over dipoles in spheres with center at the rare earth. (The number of dipoles in the summation is given in parenthesis.) The values of $\frac{4}{3} \pi \rho$ are also shown for comparison.

\begin{tabular}{lccc}
\hline \hline & $\delta_{c}\left(\mathrm{~g} / \mathrm{cm}^{3}\right)$ & $\frac{4}{3} \pi \rho\left(\mathrm{g} / \mathrm{cm}^{3}\right)$ \\
\hline $\mathrm{LiErF}_{4}$ & $10.3(920)$ & $9.6(5360)$ & 24.4 \\
$\mathrm{LiHoF}_{4}$ & $10.0(912)$ & $9.5(4604)$ & 23.9 \\
\hline \hline
\end{tabular}




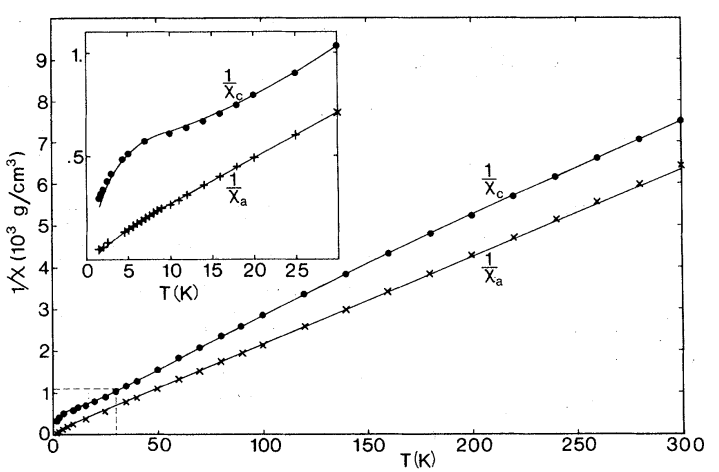

FIG. 1. $\mathrm{LiErF}_{4}$. Experimental susceptibilities $1 / \chi_{c}(T)$ $(\bullet)$ and $1 / \chi_{a}(T)(x)$. Calculated susceptibilities corresponding to the best fit with six CFP's and two EP's (solid lines).

\section{EXPERIMENTAL DETAILS}

A. Crystal growth and sample preparation

The $\mathrm{LiErF}_{4}$ and $\mathrm{LiHoF}_{4}$ single crystals were grown in this laboratory by the hydrofluorination method described by Laursen et al. ${ }^{9}$ The starting materials were of purity $>99.9 \%$. The crystals were examined by $\mathrm{x}$-ray diffraction by the rotating crystal method and the obtained cell dimensions were consistent with those given by Keller and Schmutz. ${ }^{14}$

The crystals selected for the measurements were ground into spheres of approximately $3 \mathrm{~mm}$ diam; the sphericality was better than $1 \%$. The spheres were oriented by the conventional Laue $\mathrm{x}$-ray technique.

\section{B. Magnetization measurements}

The magnetization measurements were per formed on a $5-\mathrm{Hz}$ vibrating-sample magnetometer ${ }^{\mathbf{1 5}}$ with axial pick-up coils and with a superconducting field coil, capable of supplying an axial field of 0-65 kG. The temperature range of the instrument is $1.3-300 \mathrm{~K}$. The accuracy of the instrument is $0.3 \%$.

To obtain the magnetic susceptibilities, the superconducting coil was operated in the persistent mode at a sufficiently low field for the magnetization to be linear in the field at each temperature.

The susceptibility curves seem to indicate that $\mathrm{LiHoF}_{4}$ orders at about $1.35 \mathrm{~K}$ and high-field magnetization curves were therefore recorded for this crystal in the easy direction at $1.3 \mathrm{~K}$, the lowest temperature obtainable, and at $4.2 \mathrm{~K}$.

\section{Li NMR measurements}

A self-supporting five-turn coil made of lacquered 0.5-mm-diam copper wire was placed around the sample and connected through a $0.5-\mathrm{m}$ - long coaxial line to a Robinson-type NMR spectrometer. The spectrometer frequency was kept fixed in the region $11-14 \mathrm{MHz}$ while the magnetic field was swept through the resonances and monitored with a field-locked NMR gaussmeter. A series of spectra were obtained with the magnetic field set in at least seven positions between the $c$ and the $a$ axes. The measurements were carried out at room temperature.

\section{RESULTS}

\section{A. Rare-earth data}

In Figs. 1 and 2 we show the experimental $1 / \chi_{c}(T)$ and $1 / \chi_{a}(T)$ for $\mathrm{LiErF}_{4}$ and $\mathrm{LiHoF}_{4}$, respectively.

It is seen that, whereas the largest susceptibility component is along the $a$ axis in $\operatorname{LiErF}_{4}(\Delta=1$ / $\chi_{c}-1 / \chi_{a}=1200 \mathrm{~g} / \mathrm{cm}^{3}$ at high temperature), it is along the $c$ axis in $\mathrm{LiHoF}_{4}\left(\Delta=-960 \mathrm{~g} / \mathrm{cm}^{3}\right)$ as is also the case in $\mathrm{LiTbF}_{4}\left(\Delta=-2500 \mathrm{~g} / \mathrm{cm}^{3}\right)$. The anisotropy in the susceptibility at low temperature is, however, much less for $\mathrm{LiErF}_{4}$ and $\mathrm{LiHoF}_{4}$ than for $\mathrm{LiTbF}_{4}$.

The slope of the reciprocal susceptibilities $d \chi^{-1} / d T=1 / C$ (in $\mathrm{g} / \mathrm{cm}^{3} \mathrm{~K}$ is at high temperature the same for the parallel and perpendicular component and has the value 22 for $\operatorname{LiErF}_{4}, 19$ for $\mathrm{LiHoF}_{4}$, and 20 for $\mathrm{LiTbF}_{4}$, at low temperature $1 / C_{c}=110$ and $1 / C_{a}=36$ for $\operatorname{LiErF}_{4}, 1 / C_{c}=16$ for $\mathrm{LiHoF}_{4}$, and $1 / C_{c}=7$ for $\mathrm{LiTbF}_{4}$. (Low-temperature $C_{a}$ are not defined for Ho and Tb compounds.)

Whereas $\mathrm{LiErF}_{4}$ does not order above $1.3 \mathrm{~K}$, $\mathrm{LiHoF}_{4}$ does order ferromagnetically with the $c$ axis as the easy axis at $1.30 \pm 0.05 \mathrm{~K}$, similar to $\mathrm{LiTbF}_{4}\left(T_{C}=2.86 \pm 0.03 \mathrm{~K}\right)$. The paramagnetic

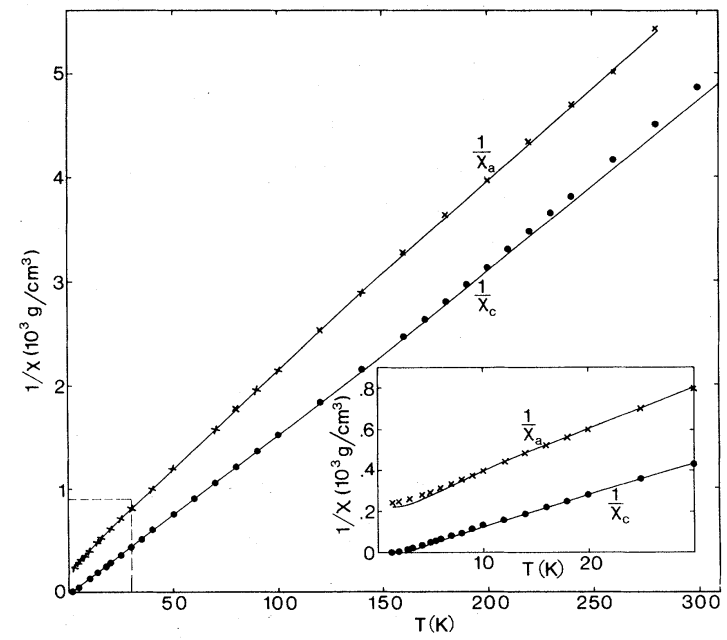

FIG。2. LiHoF $_{4}$. Experimental susceptibilities $1 / \chi_{c}(T)$ $(\bullet)$ and $1 / \chi_{a}(T)(\times)$. Calculated susceptibilities corresponding to the best fit with six CFP's and two EP's (solid lines). 


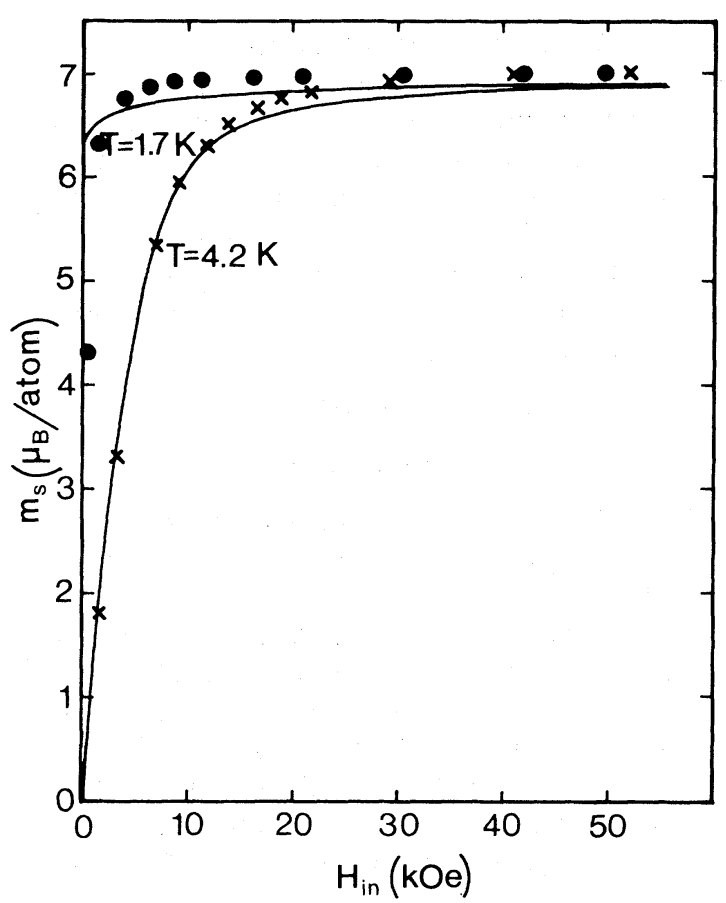

FIG. 3. $\mathrm{LiHoF}_{4}$ magnetizations vs inner magnetic field $\overrightarrow{\mathrm{H}}_{\mathrm{in}}$ for two temperatures. $T=1.3 \mathrm{~K}(\bullet), T=4.2 \mathrm{~K}$ $(\mathbf{x})$.

Curie temperatures in the easy direction are $0.5 \pm 0.3,2.0 \pm 0.2$, and $3.6 \pm 0.1 \mathrm{~K}$ for the $\mathrm{Er}$, $\mathrm{Ho}$, and $\mathrm{Tb}$ compounds, respectively.

In Fig. 3 the magnetization curves for $\mathrm{LiHoF}_{4}$ at 1.3 and $4.2 \mathrm{~K}$ are seen. The saturation moment is $6.98 \pm 0.02 \mu_{B}$ /atom. All these param- eters are collected in Table II for comparison with theory, which will be done later.

$$
\text { B. Li data }
$$

In Fig. 4 the NMR rotation spectrum of $\mathrm{Li}^{7}$ in $\mathrm{LiErF}_{4}$ is shown. It consists of three lines and from the insert, showing the actual recording for $\overrightarrow{\mathrm{H}}_{\mathrm{in}} \| \overrightarrow{\mathrm{c}}$, it is seen that the line intensities are in the ratio $3: 4: 3$. The spectra for ${ }^{7} \mathrm{Li}$ in $\mathrm{LiHoF}_{4}$ and $\mathrm{LiTbF}_{4}$ have similar appearances.

For $\overrightarrow{\mathrm{H}}_{\text {in }}$ making an angle $\theta$ with the $c$ axes the distance $\Delta \nu$ between the side lines is

$$
\Delta \nu(\theta)=\left[e^{2} q Q\left(1+\gamma_{\infty}\right) / 4 h\right](3 \cos 2 \theta+1) .
$$

Using $Q=0.1 \times 10^{-24} \mathrm{~cm}^{2},{ }^{16}$ and $\gamma_{\infty}=-0.256,{ }^{17}$ we obtain the $q$ 's displayed in Table III.

\section{DISCUSSION}

As starting CFP's for LiErF $\mathrm{L}_{4}$ in the fitting procedure the values measured on $\mathrm{LiYF}_{4}$ doped with Er were used. ${ }^{7,18}$ In order to get reasonable starting values for $\mathrm{LiHoF}_{4}$ the CFP's obtained from $\mathrm{LiYF}_{4}$ doped with a rare earth were plotted versus the number of $4 f$ electrons (Fig. 5), and interpolated values for Ho were found. The final CFP's of the four- and eight-parameter best fits together with the starting values are shown in Table IV and their standard deviations are given.

The exchange constants are found to be substantially smaller than the dipolar coupling constants in agreement with the results for $\mathrm{LiTbF}_{4} \cdot{ }^{12}$ It is seen from Table IV that the four-parameter fits are almost as good as the eight-parameter fits confirming the hypothesis that $D_{2 d}$ symmetry

TABLE II. Experimental and theoretical magnetic data for $\mathrm{LiErF}_{4}$ and

\begin{tabular}{|c|c|c|c|c|}
\hline & & $\mathrm{LiErF}_{4}$ & $\mathrm{LiHoF}_{4}$ & $\mathrm{LiTbF}_{4}$ \\
\hline High-temp. $1 / x$ anisotropy & Expt & 1200 & -960 & -2500 \\
\hline $1 / \chi_{11}-1 / \chi_{\perp}\left(\mathrm{g} / \mathrm{cm}^{3}\right)$ & Theor & 1290 & -1130 & -2340 \\
\hline \multirow{2}{*}{$\left.\begin{array}{l}\text { High-temp. } 1 / \chi \text { slope } \\
\frac{\partial \chi^{-1}}{\partial T}=\frac{1}{C^{\text {high }}}\left(\frac{\mathrm{g}}{\mathrm{cm}^{3} \mathrm{~K}}\right)\end{array}\right\}$} & Expt & 22 & 19 & 20 \\
\hline & Theor & 21.7 & 17.5 & 20.5 \\
\hline \multirow{4}{*}{$\left.\begin{array}{l}\text { Low-temp. } 1 / \chi \text { slope } \\
\frac{\partial \chi^{-1}}{\partial T}=\frac{1}{C^{10 w}}\left(\frac{\mathrm{g}}{\mathrm{cm}^{3} \mathrm{~K}}\right)\end{array}\right\} \begin{array}{l}\frac{1}{C_{c}^{1 \text { low }}} \\
\frac{1}{C_{a}^{1 \text { low }}}\end{array}$} & Expt & 110 & 16 & 8 \\
\hline & Theor & 270 & 14.6 & 8.1 \\
\hline & Expt & 36 & $\cdots$ & $\cdots$ \\
\hline & Theor & 39.6 & $\cdots$ & $\cdot$ \\
\hline Ordering temperature $T_{C}(\mathrm{~K})$ & Expt & $\cdots$ & $1.30 \pm 0.05$ & $2.86 \pm 0.08$ \\
\hline Easy axis or easy plane & & $a b$ & $c$ & $c$ \\
\hline \multirow{2}{*}{$\left.\begin{array}{l}\text { Easy-axis or easy-plane } \\
\text { Paramagnetic Curie point } \\
\Theta_{j}(\mathrm{~K})\end{array}\right\}$} & Expt & $0.5 \pm 0.3$ & $2.0 \pm 0.2$ & $3.6 \pm 0.1$ \\
\hline & Theor & 0.49 & 2.4 & 3.9 \\
\hline \multirow{2}{*}{$\left.\begin{array}{l}\text { Saturation magnetization } \\
m_{s}\left(\mu_{B} / \text { atom }\right)\end{array}\right\}$} & Expt & $\cdots$ & $6.98 \pm 0.02$ & $8.90 \pm 0.03$ \\
\hline & Theor & 4.10 & 6.88 & 8.86 \\
\hline
\end{tabular}
$\mathrm{LiHoF}_{4} \cdot \mathrm{LiTbF}_{4}$ data (Ref. 10, 11) are included for comparison. 


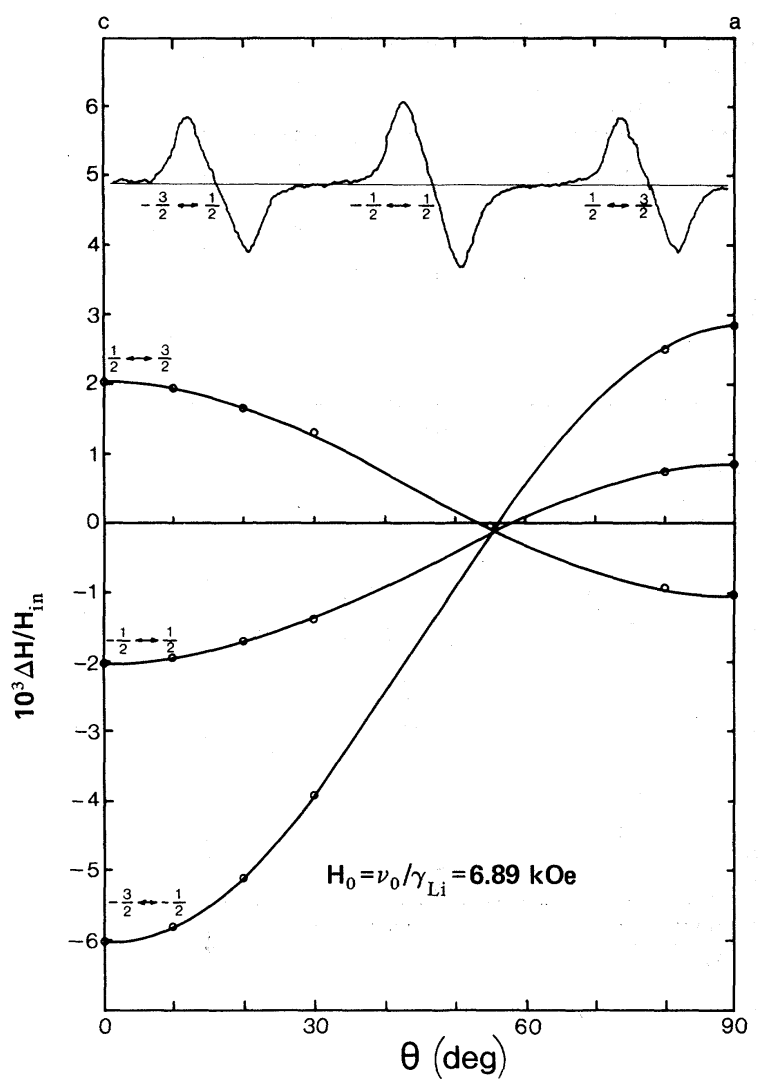

FIG. 4. ${ }^{7} \mathrm{Li}$ rotation spectrum from $\mathrm{LiErF}_{4}$ 。 The look of the recording for $\overrightarrow{\mathrm{H}}_{\mathrm{in}}$ along the $c$ axis is also shown.

is almost present. The CFP's and their accuracies are also plotted in Fig. 5. (The calculated reciprocal susceptibilities corresponding to the eight-parameter fits are also shown in Figs. 1 and 2 by the full lines.) Because of the possibility of collective short-range effects (invalidating the mean-field approximation) at low temperatures, a series of fits disregarding the lowest-temperature data (up to $6 \mathrm{~K}$ ) were performed, and the accuracy of the parameters is estimated from the variation obtained in this way. (The accuracy judged from the change in standard deviations with variation of the CFP's is much better.)

For $\mathrm{LiYF}_{4}$ doped with Er, Karayianis ${ }^{18}$ gives three sets of CFP's. We have tried all these as starting values in the fitting procedure allowing each parameter to vary only about $100 \%$. It is seen from Table $\mathrm{V}$ that our method picks out the right set. (Experience indicates that there is not a big difference between the CFP's in diluted and undiluted systems.)

The derived CFP's correspond to the energies and eigenfunctions given in Table VI for $\mathrm{LiErF}_{4}$ and in Table VII for LiHoF $_{4}$.

For $\mathrm{LiErF}_{4}$ the crystal levels, which are Kramers degenerate, are divided into two sets $E^{\prime}$ and
$E^{\prime \prime}$, transforming according to the corresponding representations of the $S_{4}$ double group. For easy reference their effective $g_{\|}$and $g_{\perp}$ are included. The ground-state $g$ values are seen to be near those derived from ESR spectra on diluted material by Sattler et al. ${ }^{8}$ who get $g_{11}=3.11$ and $g_{\perp}=8.11$.

Similarly for $\mathrm{LiHoF}_{4}$ the levels are divided into three sets, two nondegenerate sets transforming according to the $A$ and $B$ representations and one doubly degenerate set transforming according to the $E$ representation of the $S_{4}$ group. For the doubly degenerate levels the effective $g_{11}$ is included $\left(g_{\perp}=0\right)$. Also here the ground-state $g_{\| 1}$ is close to the value from the ESR spectrum of diluted material, $g_{\|}=13.3 .^{19}$

The near correspondence of the ground-state values of $g$ for the diluted and for the dense materials is not obvious, because the CFP's are different and the ground-state wave functions are rather mixed (see Tables VI and VII). This is different from the case of $\mathrm{LiTbF}_{4}$, where almost only $|6\rangle$ and $|-6\rangle$ free-ion states are involved (see the detailed discussion by Laursen and Holmes ${ }^{9}$ ).

In the higher symmetry $D_{2 d}$ the $A$ and $B$ representations split up into two representations each $\left(A_{+}, A_{-}\right.$and $\left.B_{+}, B_{-}\right)$. In the $A_{+}$and $A_{-}$representations the wave functions have the forms

$$
\sum_{n=0}^{\leqslant J / 4} a_{n}(|4 n\rangle+|-4 n\rangle)
$$

and

$$
\sum_{n=1}^{\leqslant J / 4} a_{n}(|4 n\rangle-|-4 n\rangle),
$$

whereas in $B_{+}$and $B_{-}$the forms are

$$
\sum_{n=1}^{\leqslant(2+J) / 4} a_{n}(|4 n-2\rangle+|-(4 n-2)\rangle)
$$

and

$$
\sum_{n=1}^{\leqslant(2+J) / 4} a_{n}(|4 n-2\rangle-|-(4 n-2)\rangle),
$$

where all $a_{n}$ 's are real. The remaining representations of $S_{4}$ do not split up in the $D_{2 d}$ symmetry, but the corresponding wave functions can be chosen completely real. Thus the proximity of the phases of the dominant $a_{n}$ 's indicates how near the sym-

TABLE III. Electric field gradients $q\left(\right.$ in $\left.\AA^{-3}\right)$ at the Li sites determined from ${ }^{7} \mathrm{Li}$ NMR rotation spectra and calculated using the point-charge model. (Experimental accuracy $\sim \pm 0.001$.$) Also included are their ratios and$ the similar ratios at the rare-earth sites for comparison.

\begin{tabular}{lcccc}
\hline \hline Compound & $q_{\text {theor }}$ & $q_{\text {expt }}$ & $\begin{array}{c}q_{\text {expt }} / q_{\text {theor }} \\
(\mathrm{Li} \text { site })\end{array}$ & $\begin{array}{c}B_{2 \text { expt }}^{0} / B_{2}^{0} \text { theor } \\
(R \text { site })\end{array}$ \\
\hline $\mathrm{LiErF}_{4}$ & 0.0415 & 0.0363 & 0.88 & 0.74 \\
$\mathrm{LiHoF}_{4}$ & 0.0407 & 0.0354 & 0.87 & 0.81 \\
$\mathrm{LiTbF}_{4}$ & 0.0393 & 0.0343 & 0.88 & 0.50 \\
\hline \hline
\end{tabular}



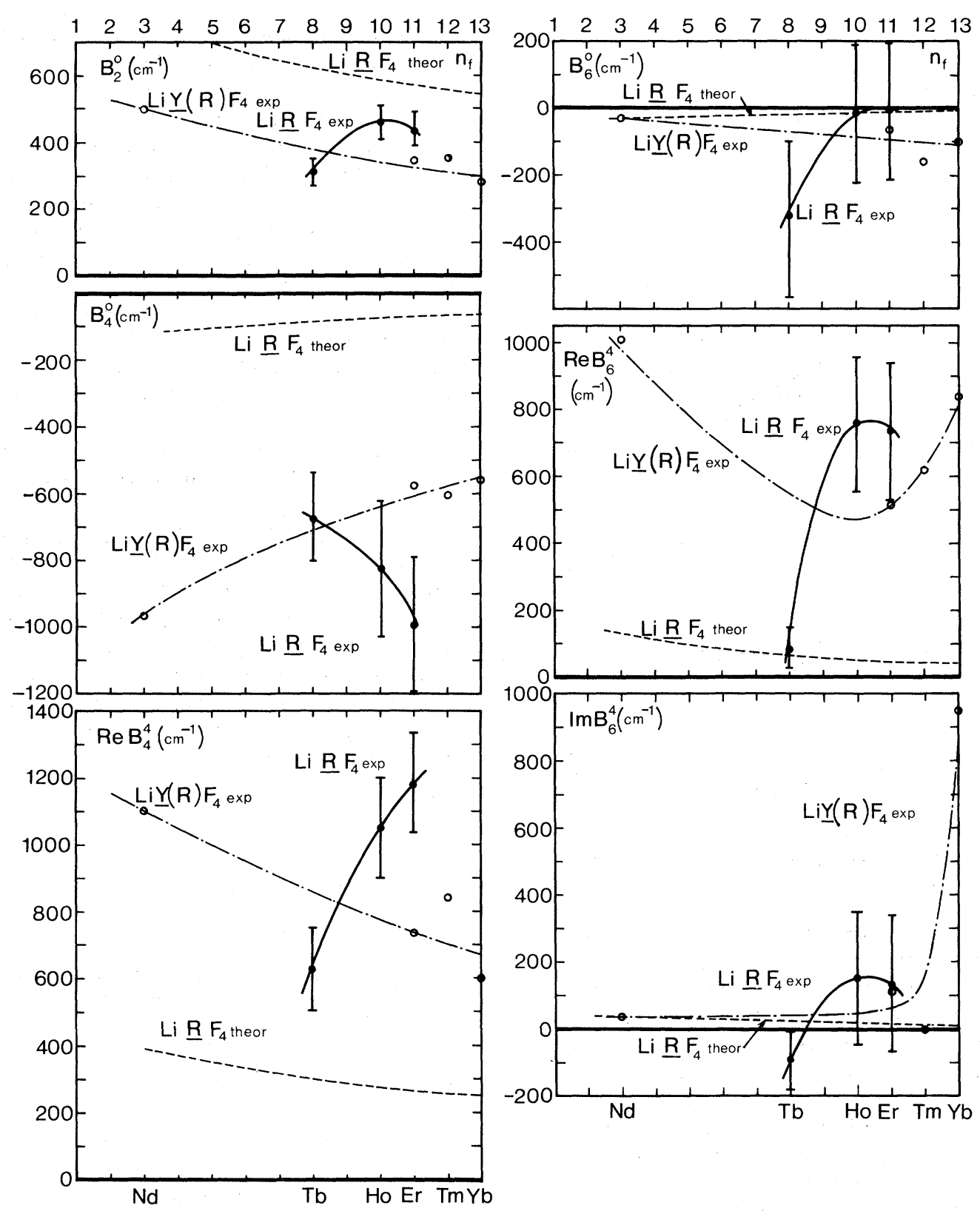

FIG. 5. O, CFP's for rare-earth-doped $\mathrm{LiYF}_{4}$ collected from Jenssen et al. (Ref. 4) (Tm), Miller and Sharp (Ref. 5) (Nd and Yb), Brown et al. (Ref. 7) (Er). •, CFP's for $\mathrm{LiErF}_{4}$ and $\mathrm{LiHoF}_{4}$ obtained in this work and for LiTbF 4 obtained by Als-Nielsen et al. (Ref. 12). The lines through the points are only for visual aid. Dashed lines are values calculated using the point-charge model.

metry through the rare-earth sites is to the $D_{2 d}$ symmetry.

The crystal energies are shown in Fig. 6 and the $\mathrm{LiTbF}_{4}$ data resulting from the CFP's of AlsNielsen et al. are also included. ${ }^{11} \mathrm{LiHoF}_{4}$ hasin contrast to $\mathrm{LiTbF}_{4}$-an exact doublet ground state. However, whereas the distance to the next level is more than $100 \mathrm{~K}$ in $\mathrm{LiTbF}_{4}$, it is only around $10 \mathrm{~K}$ in $\mathrm{LiHoF}_{4}$.

If no states existed except the ground states, the material would be an Ising system for all fields and temperatures. The third state near the ground states will limit the range of fields and temperatures for which it can be considered an Ising system.

The temperature should be so low that the third level does not become appreciably populated, i. e. , below, say, $2.5 \mathrm{~K}\left(\sim\right.$ twice $\left.T_{C}\right)$.

The allowed magnetic field strength depends on whether the field is applied along the $c$ direction or in the $a b$ plane. A $c$ field does not mix the 
TABLE IV. CFP's (in $\mathrm{cm}^{-1}$ ) at the rare-earth sites determined by fitting the susceptibility measurements either allowing to vary six CFP's and two EP's or only four CF P's fixing the remaining parameters at zero. The CFP's for $\mathrm{LiTbF}_{4}$ owing to Als-Nielsen et al. (Ref. 12) are shown for comparison. Also included are CFP's calculated from the point-charge model and their ratios to the experimental values. $S$ is the relative standard deviation.

\begin{tabular}{|c|c|c|c|c|c|c|c|c|}
\hline Compound & Remarks & $B_{2}^{0}$ & $B_{4}^{0}$ & $B_{4}^{4}$ & $B_{6}^{0}$ & $\operatorname{Re} B_{6}^{4}$ & $\operatorname{Im} B_{6}^{4}$ & $s$ \\
\hline \multirow{6}{*}{$\operatorname{LiErF}_{4}$} & Starting values & $(350)$ & $(-570)$ & $(730)$ & $(-65)$ & $(510)$ & $(120)$ & $(0.12)$ \\
\hline & $\left.\begin{array}{c}\text { Final values } \\
(6 \mathrm{CFP}+2 \mathrm{EP})\end{array}\right\}$ & 430 & -985 & 1185 & -5 & 740 & 135 & 0.010 \\
\hline & $\left.\begin{array}{c}\text { Final values } \\
(4 \mathrm{CFP})\end{array}\right\}$ & 450 & -1040 & 975 & 0 & 674 & 0 & 0.023 \\
\hline & Accuracy & \pm 50 & \pm 200 & \pm 150 & \pm 200 & \pm 200 & \pm 200 & $\cdots$ \\
\hline & $\begin{array}{c}\text { Point-charge model } \\
\text { (Theor) }\end{array}$ & 575 & -70 & 270 & -20 & 45 & 10 & $\cdots$ \\
\hline & Ratio & 0.74 & 14 & 4.4 & $(0.25)$ & 16 & (14) & $\cdots$ \\
\hline \multirow{6}{*}{$\mathrm{LiHoF}_{4}$} & Starting values & $(350)$ & $(-600)$ & $(740)$ & $(-55)$ & $(550)$ & (105) & $(0.14)$ \\
\hline & $\left.\begin{array}{c}\text { Final values } \\
(6 \mathrm{CFP}+2 \mathrm{EP})\end{array}\right\}$ & 470 & -825 & 1050 & -10 & 760 & 150 & 0.015 \\
\hline & $\left.\begin{array}{l}\text { Final values } \\
(4 \text { CF P) }\end{array}\right\}$ & 450 & -930 & 1090 & 0 & 850 & 0 & 0.015 \\
\hline & Accuracy & \pm 50 & \pm 200 & \pm 150 & \pm 200 & \pm 200 & \pm 200 & $\cdots$ \\
\hline & $\left.\begin{array}{c}\text { Point-charge model } \\
\text { (Theor.) }\end{array}\right\}$ & 585 & -75 & 280 & -22 & 50 & 10 & $\cdots$ \\
\hline & Ratio & 0.81 & 11 & 3.7 & $(0.5)$ & 15 & $(1.5)$ & $\cdots$ \\
\hline \multirow{6}{*}{$\mathrm{LiTbF}_{4}$} & CFP's & & & & & & & \\
\hline & $\begin{array}{l}\text { Als-Nielsen } \\
\quad \text { et al. }\end{array}$ & 316 & -673 & 628 & -338 & 82 & -92 & $\cdots$ \\
\hline & Accuracy & \pm 30 & \pm 130 & \pm 130 & \pm 250 & \pm 60 & \pm 60 & $\cdots$ \\
\hline & Point-charge model & & & & & & & \\
\hline & (Theor.) & 630 & -105 & 295 & -25 & 60 & 18 & $\cdots$ \\
\hline & Ratio & 0.50 & 6.4 & 2.1 & (14) & (1.4) & $(-5)$ & ... \\
\hline
\end{tabular}

ground states and the third state, and so the third state is only felt through the repopulation effect, which is negligible in the temperature range allowed.

The influence of a field in the $a b$ plane is shown in Fig. 7, where the energies of the three lowest states have been plotted versus the field and the calculated magnetic moment in the $a b$ plane has been indicated. It is seen that for fields less than, say, $5 \mathrm{kOe}$ (the local $c$ field owing to the spontaneous magnetization is around $5 \mathrm{kOe}$ ), the moment is less than $20 \%$ of the saturation moment.
Therefore for limited fields and temperatures $\mathrm{LiHoF}_{4}$ will behave quite Ising-like, but not as well as $\mathrm{LiTbF}_{4}$.

The entire crystal-field splitting $\Delta E_{\mathrm{cr}}$ is of the order of $400 \mathrm{~K}$ in $\mathrm{LiTbF}_{4}$ and $600 \mathrm{~K}$ in $\mathrm{LiHoF}_{4}$ and $\mathrm{LiErF}_{4}$.

Although experience tells that one will not find agreement between the measured CFP's and those calculated in the simple point-charge approximation, it is tempting to examine, in the case of the three materials at hand, if they display some common features. The calculated CFP's are there-

TABLE V. Three sets of CFP's given by Karayianis (Ref. 18) are used as starting values and allowed to vary not more than about $100 \%$ for best fit with the measured susceptibilities. Only set $A$ can give agreement, which is in accordance with Sattler and Nemarich (Ref. 8).

\begin{tabular}{lrrrrrrrl}
\hline \hline & & & & & & & Starting & Final \\
& $B_{2}^{0}$ & $B_{4}^{0}$ & $B_{4}^{4}$ & $B_{6}^{0}$ & $\operatorname{Re} B_{6}^{4}$ & $\operatorname{Im} B_{6}^{4}$ & $S$ & $S$ \\
\hline$A$ & 347 & -571 & 727 & -65 & 510 & 122 & 0.12 & 0.013 \\
$B$ & 190 & -1184 & 858 & -45 & 295 & 0 & 0.34 & 0.33 \\
$C$ & -230 & -1368 & 632 & 408 & 239 & 0 & 0.24 & 0.23 \\
\hline \hline
\end{tabular}


TABLE VI. Energies, wave functions, and $g$ values for the crystal levels of $\mathrm{Er}$ in $\mathrm{LiErF}_{4}$ transforming according to the $E^{\prime}$ and $E^{\prime \prime}$ representations of the $S_{4}$ double point group.

\begin{tabular}{ccccccc}
\hline \hline \multicolumn{7}{c}{$E^{\prime}$} \\
$E(\mathrm{~K})$ & $\left| \pm \frac{15}{2}\right\rangle$ & $e^{\mp i 8.7^{\circ}\left| \pm \frac{7}{2}\right\rangle}$ & $e^{ \pm i 9.6^{\circ}\left|\mp \frac{1}{2}\right\rangle}$ & $e^{ \pm i 7.7^{\circ}\left|\mp \frac{9}{2}\right\rangle}$ & $\mid g_{\|}$ & $\left|g_{\perp}\right|$ \\
\hline 517 & 0.606 & 0.734 & 0.204 & 0.229 & 10.5 & 3.2 \\
452 & 0.218 & 0.202 & -0.403 & -0.865 & 7.1 & 1.5 \\
95 & 0.474 & -0.310 & -0.728 & 0.387 & 2.6 & 3.3 \\
27 & 0.600 & -0.570 & 0.515 & -0.221 & 8.4 & 4.6 \\
& & & & & & \\
& & & $E^{\prime \prime}$ & & & \\
$E(\mathrm{~K})$ & $\left| \pm \frac{13}{2}\right\rangle$ & $e^{\mp i 7.9^{\circ}\left|\mp \frac{5}{2}\right\rangle}$ & $e^{\mp i 76.8^{\circ}\left|\mp \frac{3}{2}\right\rangle}$ & $e^{\mp i 83.1^{\circ}\left|\mp \frac{11}{2}\right\rangle}$ & $\left|g_{\mid 1}\right|$ & $\left|g_{\perp}\right|$ \\
\hline 613 & 0.753 & 0.599 & -0.169 & -0.213 & 10.3 & 3.9 \\
572 & 0.227 & 0.148 & 0.511 & 0.816 & 8.8 & 3.8 \\
44 & 0.410 & -0.487 & 0.642 & -0.428 & 0.2 & 8.0 \\
0 & 0.461 & -0.618 & -0.546 & 0.326 & 3.1 & 8.2 \\
\hline \hline
\end{tabular}

fore included in Table IV and Fig. 5. It is seen that the ratios $B_{i}^{m}(\operatorname{expt}) / B_{i}^{m}$ (theor) are very different from one and vary with $i, m$, and the rare earth involved. If the difference originated in the rare-earth filled electron shells giving rise to an isotropic shielding, the ratio would be independent of $m$ and the type of rare earth. Therefore the CFP's probably have to be calculated in the ligandfield scheme.

One interesting feature, however, emerges from the CFP's calculated in the simple point-charge approximation: The nearest neighbors to a rare earth (eight $\mathrm{F}$ ) are in positions that give the $R$ site almost $D_{2 d}$ symmetry with the fourfold axis along the crystalline $z$ axis and the twofold axes making angles of $-13^{\circ}$ with the $x$ and $y$ crystalline axes. In that " $-13^{\circ}$ rotated coordinate system" the CFP's should be almost real, if $D_{2 d}$ symmetry is almost present. Indeed for all three materials the theoretical $\operatorname{Im} B_{4}^{4} / \operatorname{Re} B_{4}^{4}$ is $<0.07$ and $\operatorname{Im} B_{6}^{4} / \operatorname{Re} B_{6}^{4}$ is $<0.21$.

We have also calculated the theoretical $\mathrm{Li}$-site CFP's using the point-charge model, and they are included in Table III with the Li experimental data. We note that they agree to within $15 \%$, and-what is more important because the $Q$ for ${ }^{7} \mathrm{Li}$ is not very exactly known-the ratio between the experimental and theoretical values does not vary significantly with the rare earth.

Thus there is a striking difference between the crystal field at the Li site and that at the $R$ site. The $\mathrm{Li}^{+}$ion is apparently a less perturbed ion than the $R^{+3}$ ion. The crystal potential just causes a slight polarization of the $\mathrm{Li}^{+} 1 s$ shell, which is simple to calculate. That $\mathrm{Li}$ is noncovalently coupled to its four surrounding F's is also suggested by the character of the long-wavelength phonon spectra found by Miller et al. ${ }^{20}$

With the crystal energy scheme approximately known, it is interesting to compare, in the highand low-temperature limit, the susceptibility and magnetization data with those which can be cal-

TABLE VII. Energies and wave functions for the crystal levels of Ho in $\mathrm{LiHoF}_{4}$ transforming according to the $A, E$, and $B$ representations of the $S_{4}$ point group. For the doubly degenerate levels ( $E$ representations) $g_{11}$ are included, $g_{\perp}=0$.

\begin{tabular}{|c|c|c|c|c|c|c|}
\hline & & & $A$ & & & \\
\hline$E(\mathrm{~K})$ & $e^{i 1.7^{\circ}}|8\rangle$ & $e^{-i 8.2^{\circ}}|4\rangle$ & $|0\rangle$ & $e^{i 8.2^{\circ}} \mid-4$ & $\left.e^{-i 1.7^{\circ} \mid}-8\right\rangle$ & \\
\hline 535 & 0.397 & -0.437 & 0.551 & -0.437 & 0.397 & $\sim A_{+}$ \\
\hline 492 & 0.530 & -0.468 & 0.000 & 0.468 & -0.530 & $\sim A_{-}$ \\
\hline 398 & -0.386 & 0.158 & 0.807 & 0.158 & -0.386 & $\sim A_{+}$ \\
\hline 92 & 0.468 & 0.530 & 0.000 & -0.530 & -0.468 & $\sim A_{.}$ \\
\hline 74 & 0.440 & 0.533 & 0.212 & 0.533 & 0.440 & $\sim A_{+}$ \\
\hline & & & $E$ & & & \\
\hline$E(\mathrm{~K})$ & $| \pm 7\rangle$ & $e^{\mp i 9.2^{\circ}}| \pm 3\rangle$ & $e^{\mp i 88 .}$ & $1^{\circ}|\mp 1\rangle$ & $e^{\mp i 92.5^{\circ}}|\mp 5\rangle$ & $\left|g_{11}\right|$ \\
\hline 546 & 0.562 & -0.717 & -0 . & .371 & 0.183 & 8.6 \\
\hline 497 & 0.281 & -0.305 & & .788 & -0.456 & 2.1 \\
\hline 124 & 0.070 & 0.023 & & .488 & 0.870 & 10.0 \\
\hline 0 & 0.775 & 0.627 & -0 & .060 & -0.045 & $\underline{13.4}$ \\
\hline & & & $B$ & & & \\
\hline$E(\mathrm{~K})$ & $e^{i 30.4^{\circ}}|6\rangle$ & $e^{i 22.7^{\circ}}|2\rangle$ & $e^{-i 2}$ & $2.7^{\circ}|-2\rangle$ & $e^{-i 30.4^{\circ}}|-6\rangle$ & \\
\hline 575 & 0.374 & -0.600 & & 0.600 & 0.374 & $\overline{\sim B_{+}}$ \\
\hline 511 & -0.423 & 0.566 & & 0.566 & 0.423 & $\sim B$ \\
\hline 39 & 0.600 & 0.374 & & 0.374 & 0.600 & $\sim B_{+}$ \\
\hline 9 & -0.566 & -0.423 & & 0.423 & 0.566 & $\sim B$ \\
\hline
\end{tabular}




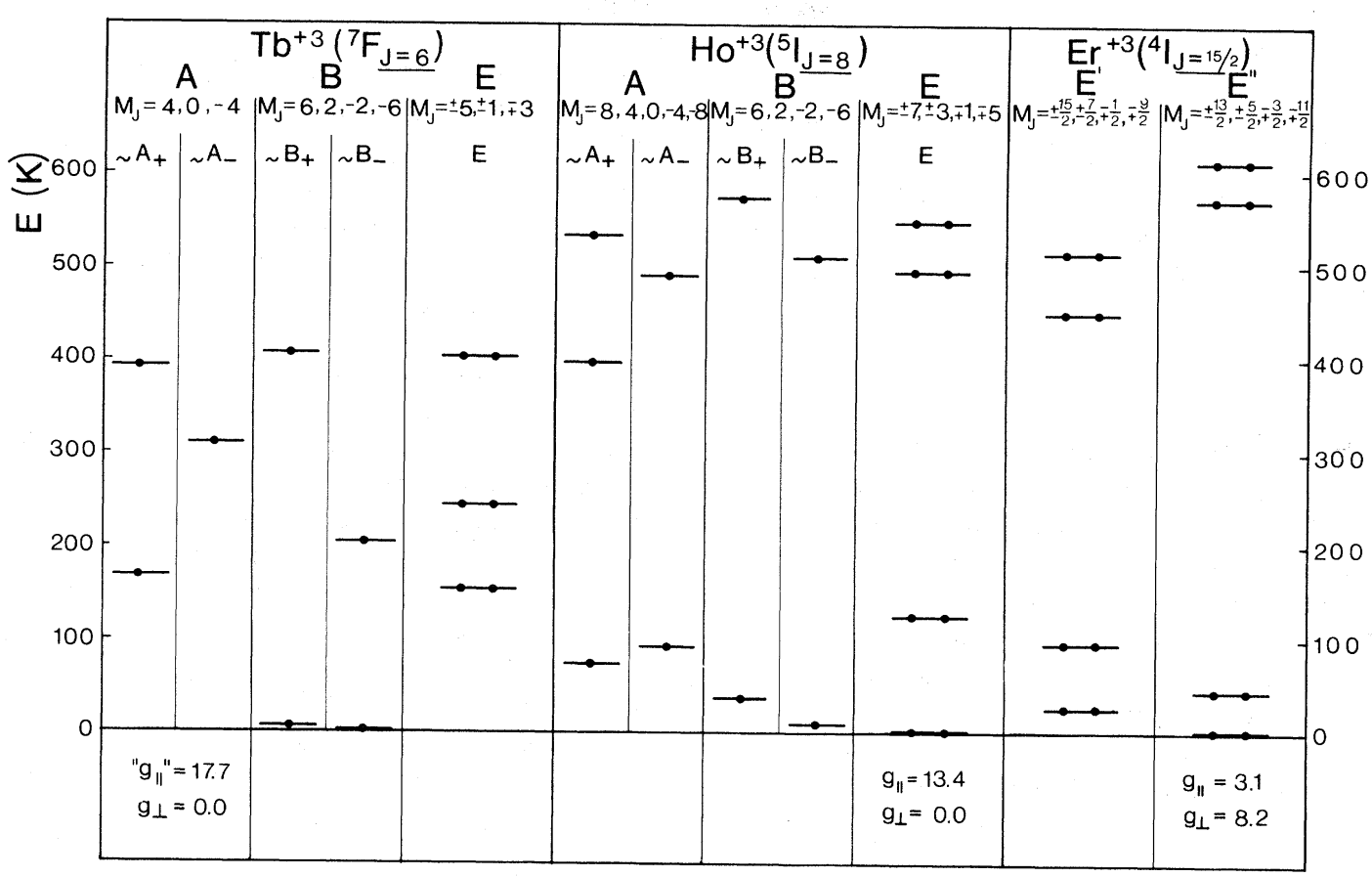

FIG. 6. Energy scheme for the crystal-field splitting of the lowest multiplet of $\mathrm{Tb}$, Ho, and Er in LiReF 4 , arranged according to representations of the $S_{4}$ point group (subgroup of $D_{2 d}$ ).

culated from the microscopic data.

The "infinite-temperature" inverse-susceptibility anisotropy depends only on $B_{2}^{0}$ and is, as shown by Frank, ${ }^{21}$

$$
\frac{1}{\chi_{c}}-\frac{1}{\chi_{a}}=\frac{9 \kappa B_{2}^{0} \beta_{J}^{2}(2 J+3)(2 J+1)}{20 \mu_{B}^{2} J(J+1) g_{J}^{2}} .
$$

The values calculated from this formula for three materials are given in Table II. It is surprising that they fit so well in spite of the fact that the assumption $k_{B} T \gg \frac{1}{2} \Delta E_{\text {cr }}$ is far from satisfied at room temperature.

Similarly the high-temperature reciprocal Curie constants

$$
\frac{1}{C}=\frac{3 k_{B} \kappa}{\mu_{B}^{2} J(J+1) g_{J}^{2}}
$$

are close to the 300-K experimental values.

The low-temperature reciprocal Curie constants

$$
\frac{1}{C_{c}}=\frac{4 k_{B} \kappa}{\mu_{B}^{2} g_{\|}^{2}} \text { and } \frac{1}{C_{a}}=\frac{4 k_{B} \kappa}{\mu_{B}^{2} g_{\perp}^{2}}
$$

are both calculated for $\mathrm{LiErF}_{4}$. For $\mathrm{LiHoF}_{4}$ and $\mathrm{LiTbF}_{4}, q_{\perp}=0$, and $1 / \chi_{a}$ goes towards the Van Vleck value for low temperature, so that only $1 / \mathrm{C}_{c}$ can be compared to experiment. Except for the $\mathrm{LiErF}{ }_{4} 1 / C_{c}$ the agreement is better than $5 \%$.

The paramagnetic Curie temperatures are

$$
\Theta_{j}=\left(g_{j}^{2} \mu_{B}^{2} / 4 k_{B}\right)\left(\frac{4}{3} \pi \rho+\delta_{j}\right) \text {, }
$$

including only dipole interaction. $j$ is either the $c$ direction or any direction in the $a b$ plane. The calculated values of the easy-direction or easyplane $\Theta_{j}$ lie only 1,15 , and $8 \%$ from the experimental values for $\mathrm{LiErF}_{4}, \mathrm{LiHoF}_{4}$, and $\mathrm{LiTbF}_{4}$, respectively, indicating that all three materials are primarily dipole coupled.

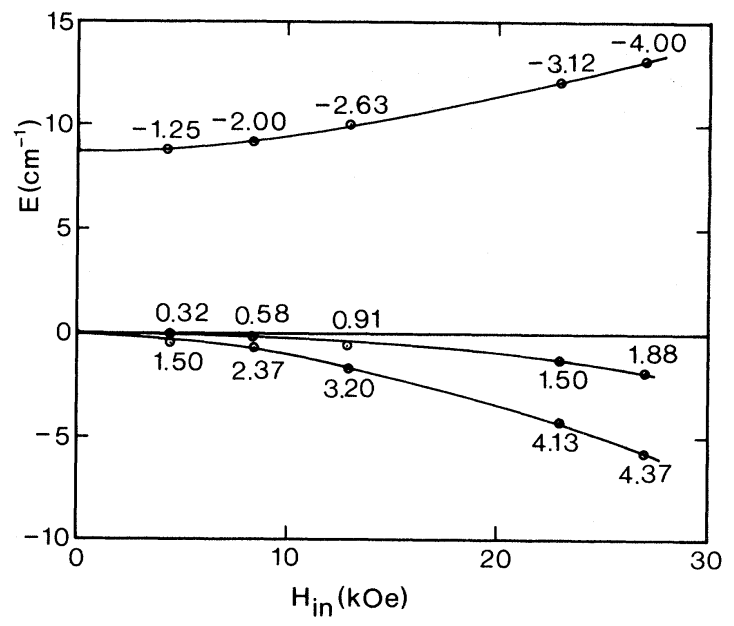

FIG. 7. Influence of a magnetic field in the $a b$ plane on the energy of the three lowest states of $\mathrm{Ho}^{3+}$ in $\mathrm{LiHoF}_{4}$. Numbers along the energy lines indicate the magnetic moments in the $a b$ plane of an ion in the corresponding state (in units of $\mu_{B}$ ). 
Finally, the saturation magnetization per ion in units of $\mu_{B}$,

$$
m_{s}=\frac{1}{2} g_{11}
$$

is calculated to be 6.88 for $\mathrm{LiHoF}_{4}$ and thus $2 \%$ lower than the experimental value. (In $\mathrm{LiTbF}_{4}$ the calculated value is only $\frac{1}{2} \%$ off the experimental one.)

\section{CONCLUSION}

$\mathrm{LiHoF}_{4}$ is found to be ferromagnetic below $1.3 \mathrm{~K}$ with the magnetic moments pointing along the $c$ axis. Furthermore, it has been shown to be a rather ideal dipolar-coupled Ising system for low magnetic fields and temperatures.

$\mathrm{LiErF}_{4}$ does not order in the considered temperature region, but extrapolation from paramagnetic data predicts that this material will order ferromagnetically below $0.5 \mathrm{~K}$, also owing to dipolar forces, with the moments in the $a b$ plane.

The magnetic characteristics in a wide temperature range (Curie temperatures, Curie constants, susceptibility anisotropies, and saturation magnetizations) of the two materials have been deter- mined and compared to those of $\mathrm{LiTbF}_{4}$.

The crystal-field parameters at the rare-earth sites have been determined from the susceptibility measurements, showing this type of measurement to be an effective way of getting approximate crystal-field parameters. The susceptibilities are especially seen to be very well suited to pick out the right set of parameters if other methods leave several alternatives.

Also, the crystal-field parameters at the Li sites have been experimentally determained. These parameters and those of the rare-earth sites are compared to values calculated using the pointcharge model, showing agreement at the Li site, but not at the rare-earth site, which indicates that in the latter case, charge transfer to the magnetic ion must be taken into account.

\section{ACKNOWLEDGMENTS}

The authors are grateful to I. Laursen for his advice concerning the growth of the crystals and for lending his crystal growth setup, and to Professor V. Frank for his valuable suggestions during the work and for his careful reading of the manuscript.
*Work partly supported by The Danish Natural Science Research Council, Grant No. 511-3575.

${ }^{1}$ R. E. Thoma, G. D. Brunton, R. A. Penneman, and T. K. Keenan, Inorg. Chem. 9, 1096 (1970).

${ }^{2}$ E. P. Chicklis and C. S. Naiman, IEEE J. Quantum Electron. QE-8, 535 (1972).

${ }^{3}$ R. K. Watts and W. C. Holton, Solid State Commun。 9, 137 (1971).

${ }^{4}$ H. P. Jenssen, A. Linz, R. P. Leavitt, C. A. Morrison, and D. E. Westman, Phys. Rev. B 11, 92 (1975).

${ }^{5}$ J. E. Miller and E. J. Sharp, J. Appl. Phys. 41, 4718 (1970).

${ }^{6}$ A. L. Harmer, A. Linz, and D. R. Gabbe, J. Phys. Chem. Solids 30,1483 (1969).

${ }^{7}$ M. R. Brown, K. G. Rooth, and W. A. Shand, J. Phys. C 2 , 593 (1969).

${ }^{8}$ J. P. Sattler and J. Nemarich, Phys. Rev. B $\underline{4}, 1$ (1971).

${ }^{9}$ I. Laursen and L. M. Holmes, J. Phys. C $\underline{7}, 3765$ (1974).
${ }^{10}$ L. M. Holmes, T. Johansson, and H. F. Guggenheim, Solid State Commun. 12, 993 (1973).

${ }^{11}$ L. M. Holmes, H. J. Guggenheim, and J. Als-Nielsen, in Proceedings of the International Conference on Magnetism (Nauka, Moscow, 1974), Vol. 6, p. 256.

${ }^{12} \mathrm{~J}$. Als-Nielsen, L. M. Holmes, and H. J. Guggenheim, Phys. Rev. Lett. 32,610 (1974).

${ }^{13}$ M. T. Hutching, Solid State Phys. 16, 227 (1964).

${ }^{14}$ C. Keller and H. Schmutz, J. Inorg。 Nucl. Chem. 27, 900 (1965).

${ }^{15} \mathrm{~T}$. Johansson (unpublished).

${ }^{16}$ Varian associates NMR table (1965) (unpublished).

${ }^{17}$ T. P. Das and R. Bersohn, Phys. Rev. 102, 733 (1956).

${ }^{18}$ N. Karayianis, J. Phys. Chem. Solids $1 \overline{97}, 2385$ (1971).

${ }^{19} \mathrm{~J}$. Tuchendler (private communication).

${ }^{20}$ S. A. Miller, H. E. Rast, and H. H. Caspers, J. Chem. Phys. 52, 4172 (1970)。

${ }^{21} \mathrm{~V}$. Frank (private communication). 